\title{
Estilos de apego parental y dependencia emocional en las relaciones románticas de una muestra de jóvenes universitarios en Colombia*
}

\section{Parental attachment styles and emotional dependence in the romantic relationships of a sample of university students in Colombia}

\author{
Brenda Liz Rocha Narváez* \\ ORCID: 0000-0001-7632-5109 \\ Jaqueline Umbarila Castiblanco \\ ORCID: 0000-0002-5937-0706 \\ Magda Meza Valencia \\ ORCID: 0000-0001-9386-7428 \\ Fabián Andrés Riveros \\ ORCID: 0000-0002-6932-4180 \\ Universidad de la Sabana, Colombia
}

Recibido: 16 enero de 2019 Revisado: 26 de febrero de 2019 Aceptado: 14 de abril de 2019

\section{Resumen}

El presente estudio tuvo como propósito identificar la relación entre los estilos de apego parental y la dependencia emocional en las relaciones románticas de un grupo de jóvenes universitarios en Colombia. Es un estudio cuantitativo de tipo descriptivo correlacional. La muestra a conveniencia fue de 500 estudiantes universitarios pertenecientes a 15 ciudades de Colombia, de la cual $34,2 \%$ fueron hombres y $65,8 \%$ mujeres entre los 18 y los 25 años. El cuestionario de recolección de datos estuvo conformado por 81 preguntas a partir de los siguientes instrumentos: datos sociodemográficos, Inventario de Apego con Padres y Pares modificado (IPPA modificado) y, Cuestionario de Dependencia Emocional (CDE). Se encuentra una correlación positiva entre los estilos de apego parental y la dependencia emocional en las relaciones románticas de los jóvenes que componen la muestra, así como una correlación positiva entre el nivel

Artículo de investigación. Cómo citar: Rocha, N. B. L., Umbarila, C. J., Meza, V. M., \& Riveros, F. A. (2019). Estilos de apego parental y dependencia emocional en las relaciones románticas de una muestra de jóvenes universitarios en Colombia. Diversitas: Perspectivas en Psicología, 15(2), 285-299. DOI: 10.15332/22563067.5065

Correspondencia: Brenda Liz Rocha, Jefe Departamento de la familia, Universidad de la Sabana. Dirección postal: Universidad de La Sabana, Campus del Puente del Común, km 7, Autopista Norte de Bogotá. Chía, Cundinamarca. Correo electrónico: brenda.rocha@unisabana.edu.co; jaquelineumca@unisabana.edu.co; magdamezavalencia@gmail.com 
socioeconómico y la variable sexo en las manifestaciones de dependencia emocional dentro de la relación romántica.

Palabras clave: apego, jóvenes, universitarios, relaciones románticas, dependencia emocional

\section{Abstract}

The purpose of the research was to identify the relationship between parental attachment styles and emotional dependence in the romantic relationships of a group of university students in Colombia. This is a quantitative study of descriptive correlational type. The sample selected, a convenience one, was 500 university students, of which $34.2 \%$ were men and $65.8 \%$ women between 18 and 25 years. The data collection questionnaire consisted of 81 questions. The questions were based on the following instruments: sociodemographic data, Parental Attachment Inventory (IPPA); Emotional Dependency Questionnaire (CDE). We found that there is a positive relationship between parental lifestyles and emotional dependence in romantic relationships, young people, the components, the sample, as well as the positive relationship between socioeconomic status and gender in the manifestations of emotional dependence within the romantic relationship.

Keywords: attachment, young people, university students, romantic relationships, emotional dependence

\section{Introducción}

Desde 1987, Hazan y Shaver determinaron que el amor romántico es un proceso de apego, mediante el cual se establecen vínculos afectivos, sociales y emocionales entre adultos, de la misma forma en que se crean vínculos afectivos entre los bebés y sus padres o cuidadores en la infancia.

Respecto al tema del apego Bowlby (1980), considerado el padre de esta teoría, afirma que los seres humanos desde el momento de su concepción hasta el día de su muerte, se encuentran expuestos a diversos modelos de interacciones afectivas con quienes los rodean; estos modelos de interacciones ayudan al individuo a percibir e interpretar las acciones e intenciones del otro y a dirigir su conducta positiva o negativamente hacia las personas con quienes tenga relaciones durante su ciclo vital. Bowlby (1995), define la teoría del apego como una forma de conducta que logra o conserva la proximidad con otro individuo identificado, a quien se considera más capacitado para enfrentar al mundo. Según el modelo de Bowlby (propuesto en 1969,1982) el individuo que enfrenta situaciones de amenaza activa el sistema de apego seguro si sus cuidadores se muestran disponibles y cercanos para atenderlo y cuidarlo; si no es así, el apego será inseguro (Martínez, Fuertes, Orgaz, Vicario, \& González, 2014). Ainsworth et al. (2015) afirman que las relaciones de apego madrehijo pueden ser medidas y clasificadas, y para esto propone la "situación del extraño". Esta es una metodología de laboratorio que le permitió clasificar las diferencias en el apego de la siguiente forma: apego seguro, apego evitativo, apego ambivalente o resistente. En cuanto al apego seguro, encontró que en la sala de juego, los niños en presencia de su madre se sentían seguros con ella y de esta manera, daban inicio a la exploración. Al ausentarse la madre de la habitación, se afectaba su capacidad de exploración y su comportamiento, hasta que volvía a entrar en contacto físico con ella y continuaba con la exploración. Ainsworth et al. (2015) observaron que la relación de estos niños con sus madres al interior del hogar era de inmensa disponibilidad y sensibilidad a los llamados de los pequeños, y calificó este patrón de conducta como saludable, ya que las madres les brindaban confianza y protección frente a la exploración. 
Con respecto al apego inseguro ambivalente, en la metodología de la situación del extraño, los estos niños exploraban con dificultad el espacio frente a la preocupación por la presencia o ausencia de sus madres. Sufrían por su ausencia, pero cuando estas regresaban dudaban en acercarse resistiéndose al contacto. La misma situación se reflejaba en los hogares, donde las madres de estos niños oscilaban entre conductas sensibles e indiferentes lo que generaba estos comportamientos inciertos en los niños.

En la situación de laboratorio, para el apego inseguro- evitativo se encontró el comportamiento de unos niños independientes de la presencia de su madre a la hora de comenzar la exploración llegando a ignorarla. Así, cuando la madre abandonaba el salón no se notaban afectados, y al regresar no variaba la conducta tratando más bien de mantenerse lejos de sus mamás. Al interior del hogar, Ainsworth et al. (2015) encontraron madres insensibles y rechazantes frente a los pedidos de los niños, lo que generaba inseguridad de parte de estos y preocupación por la proximidad. Se encontraba que ante distintas situaciones de frustración preferían asumir una posición de indiferencia frente a ellas.

Posteriormente, Main y Solomon (1990) establecieron un cuarto tipo de apego, denominado "desorganizado". Se trata de la utilización de estrategias por parte del niño que desbordan y colapsan lo que se puede calificar de apego seguro e inseguro. Lo que sucede es que el niño ante una situación de estrés (situación del extraño), incluso con la presencia de la figura de apego es para ellos fuente de temor y descontrol, generando reacciones conflictivas; muchas veces este apego se genera frente a situaciones de abandono extremo o de abuso (Soares \& Dias, 2007).

En esta línea de las interacciones afectivas, Cuervo Martínez (2010) destaca el papel de la familia como influenciadora en el proceso del desarrollo socioafectivo de los niños, lo cual se verá reflejado en sus habilidades adaptativas, sus conductas prosociales y en la regulación emocional en situaciones como el estrés, la depresión, la agresividad, la baja autoestima y la ansiedad, entre otras.

Por su parte Masota, Sedano y de Soria (2005) se refieren a la relación parental y familiar como una de las principales fuentes de sociabilidad a partir del aspecto afectivo y del estado emocional de las personas. De acuerdo con Schore (2016), las interacciones afectivas negativas a nivel parental pueden conducir a una desconfiguración a nivel cerebral, ya que durante la infancia, el cerebro se moldea significativamente y forma circuitos neuronales en la corteza orbifrontal, puesto que ésta regula la conducta social, la toma de decisiones y la inhibición de conductas (García Molina, Enseñat Cantallops, Tirapu Ustárroz, \& Roig Rovira 2009). Así, esta estructura cerebral se ve afectada al recibir la influencia de las relaciones positivas o negativas con personas cercanas, registrándose en especial las relaciones negativas. Por lo tanto, si desde la infancia se producen relaciones con características de apego inseguro, estas relaciones podrán marcar los circuitos neuronales y limitarán la capacidad para regular las emociones y como consecuencia la persona en la adultez manifestará estos mismos comportamientos de apego inseguro considerados negativos.

Dentro de las variadas definiciones que existen de las relaciones interpersonales, Salgado et al., (2016) las definen como asociaciones a largo plazo entre personas, que pueden basarse en emociones y sentimientos; por ejemplo, el amor, el interés por los negocios, las actividades sociales, las interacciones y la convivencia en el hogar, entre otras. Yepes Stork (1996) afirma que a lo largo de la vida las personas necesitan relacionarse con otros para reconocerse a sí mismos, desarrollar sus vidas con normalidad y alcanzar la plenitud en su vida afectiva. Dentro de los diferentes tipos de relaciones interpersonales se encuentran las relaciones románticas. Blandón y López (2016), las presentan como una variable importante en las expectativas de pareja que tienen los jóvenes, puesto que en este tipo de relaciones se conjugan aspectos que trascienden más allá de la atracción y del contacto físico. Por tanto, es importante estudiar la calidad de las relaciones afectivas entre los jóvenes, ya que estas se ven afectadas por variables como los celos, la violencia, la idealización del amor y el control exagerado, entre otras, sobre las cuales se debe intervenir asertivamente, con herramientas y conocimientos, dirigidos a fortalecer tanto comportamiento como actitudes saludables dentro de las 
relaciones románticas (Hernando-Gómez, MaraverLópez, \& Pazos-Gómez, 2016).

Martínez et al. (2014) al referirse al ajuste y calidad de las relaciones de pareja de jóvenes adultos, resaltan la importancia de reconocer el papel que juegan los procesos afectivos de la infancia, y a su vez, abordan la conexión entre el apego en la infancia, el apego en las relaciones de jóvenes adultos y la calidad de estas. Furman y Collibee (2018) afirman que las relaciones con los padres pueden ser la base para tener intimidad con los demás, e igualmente, los amigos aportan al desarrollo de las relaciones de intimidad, del apego y del cuidado mutuo, aspectos fundamentales en las relaciones románticas.

Dentro de las características de las relaciones románticas se encuentran: la colaboración, la reciprocidad, la solidaridad, la co-construcción, la afiliación, el mutuo cuidado y la atracción sexual (Furman \& Simón, 1999). Estas características al ser adecuadas son un instrumento para favorecer el cambio de actitudes en positivo; por lo tanto, son fundamentales para tener una buena calidad de vida, una sociedad sana y una buena educación sentimental (Callejón-Chinchilla \& Gila-Ordoñez, 2018).

Castelló Blasco (2013) afirma que, si los rasgos de colaboración, reciprocidad, solidaridad, co-construcción, afiliación, mutuo cuidado y atracción sexual, dejan de ser adaptativos, frecuentes y deseables en las relaciones románticas, y se manifiestan de una forma exagerada, podrían conducir a una dependencia emocional. Cabe subrayar, que la diferencia entre las relaciones románticas que presentan dependencia emocional y las que no la presentan está en "la magnitud de todos los sentimientos y comportamientos implicados” (p.172). Así mismo, Jorge Castelló Blasco, considera que la dependencia emocional es "un patrón crónico de demandas afectivas frustradas, que buscan desesperadamente satisfacerse mediante relaciones interpersonales estrechas" (2000, p.7), y afirma que, para las personas dependientes emocionalmente, el amor es lo más relevante, pues viven sus relaciones intensamente, donde lo que más les importa es que la otra persona esté a su lado, y por lo tanto, desarrollan relaciones de pareja desequilibradas, ya que el dependiente emocional aporta más que el otro (Castelló Blasco, 2012).

La dependencia emocional, se caracteriza por el énfasis extremo de tipo afectivo que una persona siente hacia otra dentro de una relación, generado por un apego ansioso y en donde persiste un fuerte deseo de intimidad con su pareja (Castelló Blasco, 2005).

Moral, García, Cuetos y Sirvent (2017) destacan que en la población de jóvenes, la dependencia emocional representa una problemática vinculada a la violencia en el noviazgo. Esta violencia, se manifiesta con el maltrato físico, maltrato emocional y con conductas de abuso; lo que indica, que los jóvenes victimizados en esas relaciones presentan mayor dependencia emocional, la cual se muestra principalmente con comportamientos de sumisión y subordinación. Estos comportamientos, son dados por la interrelación de factores como el apego patológico, los procesos de autoengaño, acomodación y manipulación relacional. Al respecto, Moral et al. (2018) encontraron en su trabajo, que los dependientes emocionales presentan antecedentes familiares como interdependencia, autoengaño, adicciones, sentimientos negativos y acomodación situacional.

En cuanto a la incidencia que tiene el apego parental y la dependencia emocional en el desarrollo de las relaciones dentro del grupo familiar, Guzmán y Contreras (2012), concluyeron que las personas con estilo de apego seguro son las que reportan los niveles de satisfacción marital más altos y las personas con estilos de apego inseguro-evitativo, los más bajos. Machado, Dias-da-Costa y da Silva (2015), muestran en su estudio que la relación entre el apego parental y el apego amoroso tiene valores significativos y que existen relaciones significativas entre el apego parental y la satisfacción con la vida, en las dimensiones de comunicación y de confianza. Igualmente, Lozano-Sapuy, Albarracín-Ángel y Vásquez-Amézquita (2016) encontraron relación entre el apego parental, el apego adulto romántico y la dependencia afectiva, en personas entre los 18 y 40 años, y concluyeron que la madre regula la manera en que se relacionan los individuos a futuro y el control del padre dosifica la evitación hacia la pareja. 
De acuerdo con lo anterior, el interés de este estudio se fundamenta en la importancia de cubrir el vacío existente en la temática de los comportamientos que se manifiestan en los diferentes estilos de apego y su correlación con los comportamientos que se manifiestan en la dependencia emocional, mencionados en párrafos anteriores, en una muestra amplia y homogénea de jóvenes universitarios en Colombia, como sugieren Acosta, Amaya y De la Espriella (2013), ya que los estudios encontrados en Colombia, se aplicaron en Bogotá, Ibagué y Medellín, con muestras más pequeñas. Debido a lo expuesto, este estudio tuvo como objetivos: Establecer los estilos de apego parental; describir las manifestaciones de dependencia emocional y su relación con sexo y el nivel socioeconómico; y, determinar la relación entre el estilo de apego parental y la dependencia emocional de los jóvenes universitarios que componen la muestra.

\section{Método}

\section{Tipo de estudio}

El presente estudio utilizó un enfoque cuantitativo, con un alcance de tipo descriptivo correlacional cuyo propósito fue "conocer la relación o grado de asociación que existe entre dos o más conceptos, categorías o variables en un contexto en particular" Hernández, Fernández y Baptista (2014, p. 93). Se analizó la asociación de los estilos de apego parental y la dependencia emocional en las relaciones románticas de una muestra de jóvenes universitarios en Colombia.

\section{Participantes}

La muestra estuvo conformada por un total de 500 estudiantes universitarios de 15 ciudades de Colombia, los cuales fueron seleccionados por conveniencia, ya que la elección dependió del criterio de los investigadores a través de un método no probabilístico (Hernández et al., 2014). Los participantes fueron 171 hombres, $(34,20 \%)$ y 329 mujeres $(65,80 \%)$, entre los 18 y los 25 años cuya edad promedio fue de 23,75 años. Respecto al tipo de instituciones de educación superior, un 16,2\% (81 estudiantes) pertenecían a instituciones públicas y un $83,8 \%$ (419 estudiantes) a instituciones privadas. El nivel socioeconómico correspondió a: bajo 107 $(21,4 \%)$, medio $298(59,6 \%)$ y alto $95(19 \%)$.

Los criterios de inclusión para los participantes fueron: tener una edad comprendida entre los 18 años y los 25 años, ser soltero y estar o haber estado vinculado en una relación romántica con una duración mínima de 6 meses (ver tabla 1).

Tabla 1.

Caracterización de la muestra

\begin{tabular}{llcc}
\hline & & Número & Porcentaje \\
\hline \multirow{2}{*}{ Sexo } & Hombre & 171 & 34.20 \\
& Mujer & 329 & 65.80 \\
\hline Institución & Pública & 81 & 16.20 \\
educativa & Privada & 419 & 83.80 \\
\hline \multirow{2}{*}{$\begin{array}{l}\text { Nivel socioe- } \\
\text { conómico }\end{array}$} & Bajo & 107 & 21.40 \\
& Medio & 298 & 59.60 \\
& Alto & 95 & 19.00 \\
\hline
\end{tabular}

Fuente: elaboración propia.

\section{Instrumentos}

La recolección de datos se hizo a través de un cuestionario conformado por 81 preguntas a partir de los siguientes instrumentos: datos sociodemográficos, Inventario de Apego con Padres y Pares modificado (IPPA-M) y Cuestionario de Dependencia Emocional (CDE).

\section{Cuestionario sociodemográfico.}

Las preguntas del cuestionario sociodemográfico estuvieron dirigidas a indagar en los participantes, el sexo, la edad, el nivel socioeconómico y el estado civil.

\section{Inventario de Apego con Padres y Pares modificado (IPPA-M, Pardo, Pineda, Carrillo, \& Castro, 2006).}

Se optó por utilizar el instrumento IPPA-M (Pardo et al., 2006), con las dos dimensiones de alienación y comunicación y confianza, que se realizó a partir 
de la propuesta "Implicaciones psicométricas de la versión española del Inventario de Apego a Padres y Pares (IPPA, Armsden \& Greenberg, 1987). Para la identificación de los perfiles de apegos reportados por Ainsworth et al. (2015), y Vivona (2000), quienes proponen tres tipos de apego: seguro, inseguro evitativo e inseguro ambivalente, se generaron los puntajes ponderados a partir del análisis confirmatorio de factores para cada uno de los participantes. Este análisis confirmatorio de factores del instrumento de 15 ítems arrojó valores adecuados de ajuste: SRMR (raíz del cuadrado medio residual estandarizado) $=0.0588$; RMSEA (error cuadrático medio de la aproximación) $=0.0824$; IC RMSEA $90 \%=$ [0.0741-0.099], CFI (índice del ajuste compartido) $=0.9203$, contra la versión original de $S R M R=0.0698$; RMSEA $=0.0735$; IC RMSEA $90 \%=$ [0.0686-0.0783], $\mathrm{CFI}=0.8794$. Donde, además, se observaron problemas de validez convergente en parámetros con probabilidades superiores al $1 \%$ y correlaciones altas entre los factores de comunicación y confianza $(r=0.87$ con IC $90 \%=[0.83-0.9])$.

El instrumento midió la calidad del apego parental percibido por los jóvenes de la muestra. Estuvo conformado por 25 ítems para cada padre, con una escala tipo Likert de cuatro puntos, donde 1 correspondía a "total acuerdo" y 4 a "total desacuerdo", con un alfa de Cronbach entre .7 y .9. Categorización: apego seguro, apego inseguro ambivalente, apego inseguro evitativo.

\section{Cuestionario de Dependencia Emocional (CDE Lemos Hoyos \& Londoño Arredondo, 2006).}

Este cuestionario consta de 23 ítems y 6 factores, con una escala tipo Likert desde 1 (completamente falso en mí) hasta 6 (me describe perfectamente). Busca medir las diferentes dimensiones de la dependencia emocional en los jóvenes de la muestra. Categorización: ansiedad de separación, expresión afectiva de la pareja, modificación de planes, miedo a la soledad, expresión límite y búsqueda de atención.

\section{Procedimiento y Análisis de datos}

Antes de aplicar en la muestra total de estudiantes el cuestionario de 81 preguntas, que contenía los tres instrumentos mencionados, se realizó una prueba con este cuestionario en 10 estudiantes universitarios, para determinar si entendían las preguntas y las escalas de respuestas. Posteriormente, se aplicó el cuestionario, de forma autoadministra$\mathrm{da}$, en 15 ciudades colombianas de 4 regiones del país y se realizó la recolección de datos a través de la aplicación Google-Docs, aplicando los principios de ética y responsabilidad para este tipo de estudios, para no generar daños o riesgos a los participantes. Posteriormente, se realizó el análisis de estos de acuerdo con los tres objetivos del estudio.

Para establecer los estilos de apego parental de la muestra se aplicaron técnicas de conglomerados de k-medias, en donde se asignaron todos los participantes a uno de los tres tipos de apegos posibles.

Para relacionar el sexo y el nivel socioeconómico y el perfil de apego, se utilizó la técnica no paramétrica de Kruskal Wallis y pruebas de comparación de medias de Tukey aplicadas a los rangos de las variables de respuesta emocional, siempre y cuando se presentaran efectos significativos de los factores evaluados al nivel de $p<.05$.

Para describir las manifestaciones de dependencia emocional en las relaciones románticas del grupo de jóvenes, se utilizó lo que ya estaba teorizado en la validación del instrumento con el que se midió la dependencia emocional, por Lemos Hoyos y Londoño Arredondo (2006) así: factor 1, ansiedad de separación: se describen las expresiones emocionales del miedo que se producen ante la posibilidad de disolución de la relación; factor 2 , expresión afectiva: necesidad del sujeto de tener constantes expresiones de afecto de su pareja que reafirmen el amor que se sienten y que calme la sensación de inseguridad; factor 3, modificación de planes: el cambio de actividades, planes y comportamientos debido a los deseos implícitos o explícitos por satisfacer a la pareja o a la simple posibilidad de compartir mayor tiempo con ella; factor 4, miedo a la soledad: dentro de la descripción del componente, se identifica el temor por no tener una relación de pareja, o por sentir que no es amado; factor 5 , 
expresión límite: la posible ruptura de una relación para el dependiente emocional puede ser algo tan catastrófico por su enfrentamiento con la soledad y la pérdida del sentido de vida, que puede llevar a que el sujeto realice acciones y manifieste expresiones impulsivas de autoagresión, relacionadas con las características de la persona con un trastorno límite de la personalidad; y factor 6 , búsqueda de atención: se tiende a la búsqueda activa de atención de la pareja para asegurar su permanencia en la relación y tratar de ser el centro en la vida de éste(a). (p. 136-137)

Para describir las manifestaciones de dependencia emocional en las relaciones románticas y su relación con sexo y nivel socio económico del grupo de jóvenes, se tomaron las variables: dependencia emocional versus sexo y nivel socioeconómico y se establecieron los promedios de las respectivas comparaciones entre las mismas variables a través de la prueba de Tukey.

Finalmente, la relación entre el estilo de apego parental y la dependencia emocional de los jóvenes, se obtuvo a través de la prueba de Kruskal-Wallis, de las dimensiones de dependencia emocional y el apego.

\section{Resultados}

\section{Estilos de apego parental}

El apego seguro puntuó con el $46.60 \%$, en su mayoría expresado por las mujeres $(60.52 \%)$ con relación a los hombres (39.48\%). Respecto al apego seguro y el nivel socioeconómico, en su mayoría se constató en jóvenes de nivel socioeconómico medio $(58.80 \%)$. Igualmente, se obtuvieron los mayores porcentajes en este mismo nivel para el apego ambivalente $(65.15 \%$ ) y el apego evitativo (55.56\%).

El restante de la población, $53.40 \%$, puntuó con indicadores de apego inseguro. Así, el $27 \%$ de esta población puntuó para un estilo de apego evitativo y $26.40 \%$ en un estilo de apego ambivalente. Las mujeres puntuaron con porcentajes altos en ambos componentes, es decir, $62.12 \%$ ambivalentes $y$ $78.52 \%$ evitativas (ver tabla 2 ).

\section{Manifestaciones de dependencia emocional y su relación con sexo y nivel socioeconómico.}

La población objeto de estudio, en las dimensiones ansiedad de separación, expresión afectiva de la pareja, modificación de planes, miedo a la soledad y expresión límite, se comportó de manera similar, sin presentar diferencias significativas en las manifestaciones de dependencia emocional, tanto en hombres como en mujeres de los diferentes niveles socioeconómicos.

En la dimensión búsqueda de atención se encontró que los hombres de nivel socioeconómico bajo presentaron las calificaciones más altas en las manifestaciones de dependencia emocional en sus relaciones románticas, seguidos por los hombres correspondientes al nivel socioeconómico medio; y finalmente los hombres de nivel socioeconómi-

Tabla 2.

Estilos de apego, nivel socioeconómico y sexo

\begin{tabular}{llccc}
\hline & & Apego seguro & Apego ambivalente & Apego evitativo \\
\hline Total & & $233(46.60 \%)$ & $132(26.40 \%)$ & $135(27.00 \%)$ \\
\hline \multirow{2}{*}{ Sexo } & Hombre & $92(39.485)$ & $50(38.88 \%)$ & $29(21.48 \%)$ \\
& Mujer & $141(60.52 \%)$ & $82(61.12 \%)$ & $106(78.52 \%)$ \\
\hline \multirow{3}{*}{ Nivel Socioeconómico } & Medio & $50(21.46 \%)$ & $27(20.46 \%)$ & $30(22.22 \%)$ \\
& Alto & $137(58.80 \%)$ & $86(65.15 \%)$ & $75(55.56 \%)$ \\
& & $46(19.74 \%)$ & $19(14.39 \%)$ & $30(22.22 \%)$ \\
\hline
\end{tabular}

Fuente: elaboración propia 
Puntaje del factor búsqueda de atención

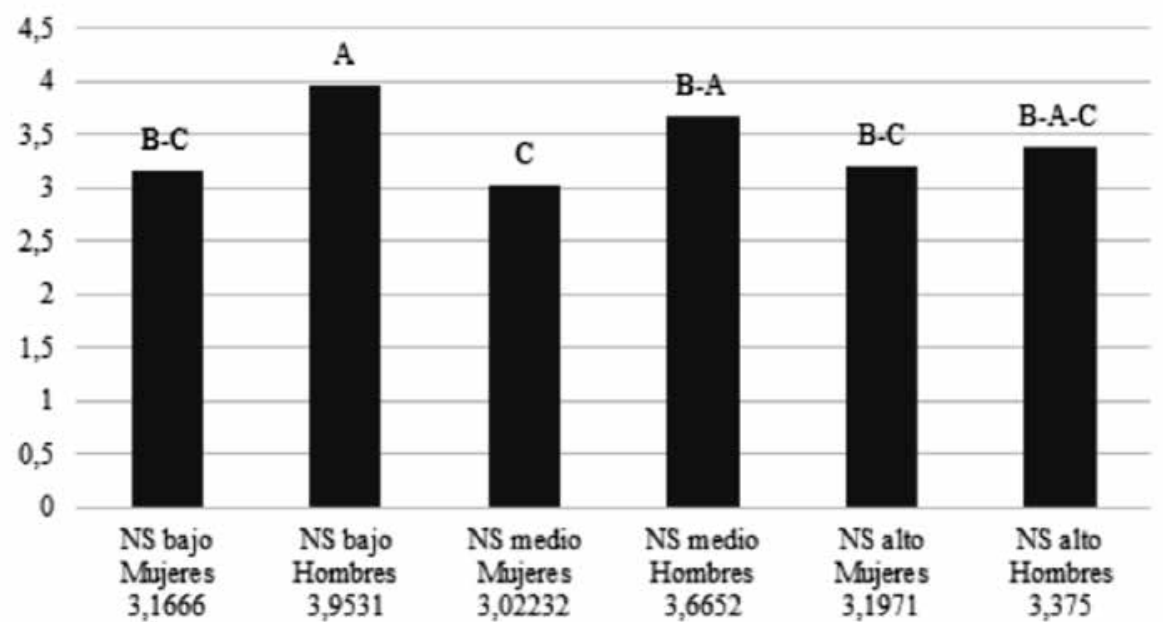

Figura 1. Dimensión de búsqueda de atención y sexo- nivel socioeconómico. Promedios con la misma letra son estadísticamente iguales bajo prueba de Tukey aplicada a rangos, $\mathrm{p}<.05$.

Fuente: elaboración propia.

co alto. Se encontró también que, a mayor nivel socioeconómico las manifestaciones de búsqueda de atención disminuyen. En los niveles socioeconómicos bajo y medio los hombres manifiestan una tendencia más alta en cuanto a la dimensión de búsqueda de atención con relación a las mujeres de los mismos niveles socioeconómicos (ver figura1).

\section{Relación entre los estilos de apego parental y la dependencia emocional.}

En todas las dimensiones de dependencia emocional se presentaron diferencias en contra de los perfiles de apego seguro. En algunas dimensiones se notan valores significativos hacia el apego inseguro ambivalente; el apego inseguro evitativo se encontró en valores intermedios, y el apego seguro siempre se presentó con valores bajos en las diferentes dimensiones de dependencia emocional (ver fig. 2).

\section{Discusión}

El objetivo principal del presente estudio fue determinar si existe una correlación entre los estilos de apego parental y la dependencia emocional en jóvenes universitarios en Colombia. En efecto, se encontró una correlación positiva entre estas dos variables. A partir del objetivo principal se derivaron otros tres objetivos y se pudo determinar lo siguiente. En cuanto al primer objetivo en donde se buscaba establecer los estilos de apego parental, se evidenció que prevaleció el apego seguro; en su mayoría manifestado por las mujeres. En este sentido, Valle y Moral (2018) hallaron que los jóvenes entre 22 y 25 años mostraron un estilo de apego seguro, al igual que Martínez, Castañeiras y Posada (2011), quienes afirman que en su investigación predominó el estilo vínculo óptimo (alto afecto y bajo control), probablemente porque coincide con el mismo grupo etario (18-25 años).

Autores como Oliva (2004), mencionan que si en la infancia se tuvo un apego seguro con los padres o con otras personas con alto significado que mostraran sensibilidad y consciencia, en su vida posterior la persona tendrá una actitud de confianza con las personas que se relacione. Al analizar la variable de dependencia emocional, es factible corroborar lo que autores como Feeney y Noller (1990) afirman en cuanto a que el apego seguro, es una base para que se den relaciones amorosas más confiables, satisfactorias y estables. De acuerdo con Sroufe (2000) son las relaciones tempranas de apego las 


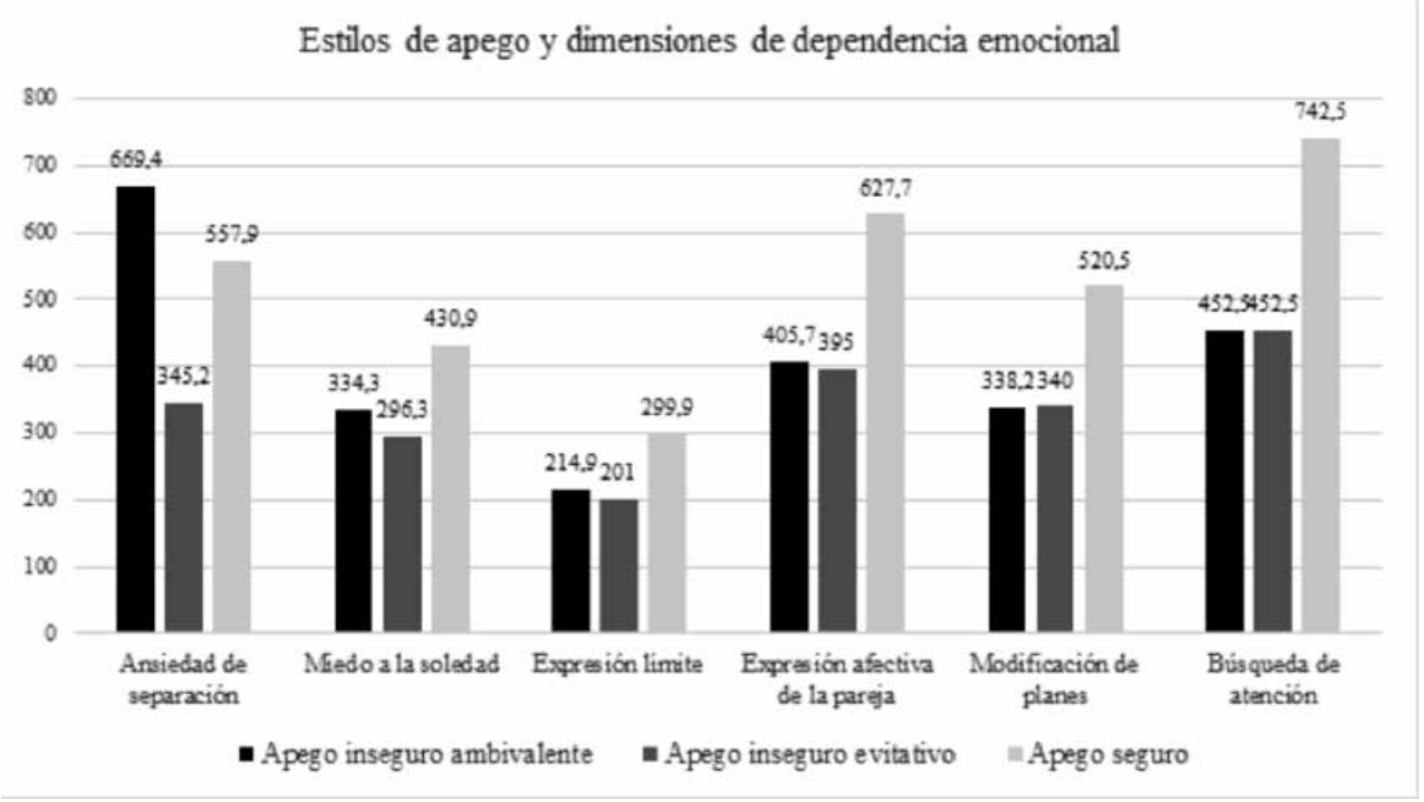

Figura 2. Dimensiones de dependencia emocional y estilos de apego

Fuente: elaboración propia.

que se pueden constituir en un prototipo de las relaciones más vinculantes que el individuo puede conformar en el transcurso de la vida. Bowlby (1995) se refiere a este proceso como la forma en que los niños infieren expectativas sobre los probables comportamientos de los demás y de ellos mismos en sus relaciones.

Con respecto a los hallazgos en los estilos de apego y los niveles socioeconómicos, se podrían explicar que al enfrentarse a mayores eventos estresantes y a un nivel de escolaridad bajo. Los padres pueden disminuir su calidad relacional con sus hijos al no poder prestar la necesaria atención y reflexión a las demandas propias del desarrollo de estos, lo cual, genera dificultades en la relación con sus cuidadores, evidenciando que el apego seguro está más afectado en los niveles socioeconómicos más bajos (Torres \& Rodrigo, 2014), lo cual se constituye en la base fundamental para lograr modelos representacionales internos que determinan la manera de percibir e interpretar las relaciones con los demás (Bowlby, 1980).

Por otro lado, se destaca en este estudio que el resto de la población puntuó con indicadores de apego inseguro. Así, el $27 \%$ de los participantes puntuaron para un apego evitativo y el $26.40 \%$ para un apego ambivalente. Las mujeres sobresalieron con porcentajes altos en ambos componentes $(62.12 \%$ ambivalentes y $78.52 \%$ evitativas). Se puede explicar la preponderancia de la población femenina con apego ansioso al acudir a los diferentes roles de género establecidos socialmente (Carreras, Brizzio, González, Mele, \& Casullo, 2008).

Estos hallazgos coinciden con lo encontrado por Gómez Maquet et al., (2009) quienes puntúan para lazo paterno y materno altos niveles de constricción cariñosa y control sin afecto de las mujeres pertenecientes a la muestra. Aunque su estudio difiere en el rango de edad utilizado (16-46 años).

Igualmente, en el estudio realizado por LozanoSapuy et al. (2016) se evidencia que la población estudiada presentó bajos niveles de afecto y altos de control, los cuales son características del apego inseguro ambivalente. No obstante, no distinguen los resultados por sexo y se observa que la mayoría de estudiantes que compusieron la muestra fueron mujeres. Lo que sugiere que pudo haber en la infancia uso de amenazas de abandono como medio de control, por lo cual, habrían manifestado una tendencia para aferrarse a sus padres o cuidadores 
al presentar exploración ansiosa y apego ambivalente; o bien, fueron rechazadas por sus padres cuando buscaron protección y mostraron apego evitativo (Reyes \& Reidl, 2015).

En cuanto al segundo objetivo de la investigación, acerca de las manifestaciones de dependencia emocional en las relaciones románticas y su relación con sexo y nivel socio económico del grupo de jóvenes universitarios, los hallazgos muestran que en los niveles socioeconómicos bajo, medio y alto, los hombres manifiestan una tendencia más alta en cuanto a la dimensión de búsqueda de atención, con relación a las mujeres de los mismos niveles. Esto indica una manifestación por ser el centro de atención y así asegurar su permanencia en la relación. De ahí que, esto sea coherente con un reciente estudio realizado en población mexicana, en una muestra de 200 personas con un rango de edad entre los 18 y los 35 años por Laca Arocena y Mejía Ceballos (2017), en donde encontraron similitudes con relación al ítem de búsqueda de atención similar a los hallazgos obtenidos en este estudio. Se identificaron también puntuaciones más altas en los hombres que en las mujeres en las dimensiones de búsqueda de atención y ansiedad por la separación. En cuanto a la búsqueda de atención de la pareja en la población mexicana, los autores relacionan el resultado directamente con el rol que el hombre desempeña en esta sociedad, en donde es él, quien ejerce el papel protagónico en la pareja, es decir, es quien habla y actúa en nombre de la pareja, lo que supone atraer su atención constantemente.

En este mismo sentido, en el estudio de Lemos, Jaller, González, Díaz y De la Ossa (2012) acerca del perfil cognitivo de dependencia emocional en jóvenes universitarios en Medellín, encontraron que el bajo desarrollo de estrategias de afrontamiento de autonomía, se evidencian comportamientos de aferramiento hacia la pareja y búsqueda de atención, al punto de hacer que su vida gire en torno a ésta al dejar de lado sus propios planes y necesidades. Si bien no se establece una diferencia con relación al sexo, sí se establece que, quien presenta este patrón de comportamiento se aleja notoriamente de la autonomía. Por lo anterior, la búsqueda de atención hace parte de las conductas auto-derrotistas que llevan al individuo a sabo- tear sus propias posibilidades de realización. Esto conlleva además a validar una vez más lo afirmado por Castelló Blasco (2005) cuando describe que las personas con dependencia emocional generalmente son débiles afectivamente y pueden sentirse necesitadas de protección. Figueroa y Franzoni (2011) a su vez, sostienen que el nivel socioeconómico, la escolaridad, el reconocimiento social, el lugar de residencia, la generación de pertenencia y las características de la familia de origen, configuran el significado y la forma de vivir la masculinidad.

De acuerdo con lo anterior, los hallazgos de este estudio se pueden relacionar con la teoría propuesta por Castelló Blasco (2005) quien afirma que, existe un estilo de dependencia para mujeres y otro para hombres, además, señala que los hombres expresan especialmente un tipo de dependencia emocional dominante con respecto a las mujeres, por cuanto estos poseen baja habilidad para regular su expresión de afecto y por su tendencia hacia la dominación, en lugar de sumisión, la cual es expresada muchas veces en actitudes y comportamientos de maltrato. A su vez afirma, que el comportamiento de los maltratadores -en su mayoría hombres- está relacionado con normas culturales machistas, en sociedades que aún consideran a la mujer como persona-objeto, para demostrar su poder o descargar todo tipo de frustraciones.

Al tener en cuenta los resultados del presente estudio y la teoría de Castelló sobre dependencia emocional dominante, se puede inferir que la tendencia a la violencia intrafamiliar en poblaciones de nivel socioeconómico bajo es mucho más alta que en otros niveles socioeconómicos.

Finalmente, el tercer objetivo de esta investigación fue identificar la relación entre el estilo de apego parental y la dependencia emocional en las relaciones románticas de la muestra. Los resultados concuerdan con los del estudio realizado por Lozano Sapuy et al. (2016), en donde encontraron que todos los factores de dependencia emocional estuvieron directamente relacionados con la ansiedad experimentada (apego inseguro) hacia la pareja. Del mismo modo, Valle y Moral (2018) afirman que existe una relación entre dependencia emocional y estilos de apego, en el sentido de que el estilo 
seguro se asocia con la ausencia de dependencia emocional. De esta manera, una historia de apego seguro podría actuar como factor de protección frente a relaciones afectivas desequilibradas en el futuro, según Smith Etxeberría et al. (2014).

Quiñones y Wilson (2013) utilizaron el cuestionario de dependencia emocional de Lemos Hoyos y Londoño Arredondo (2006), para evaluar el nivel de dependencia emocional en una muestra de adolescentes bogotanos y concluyeron que no existía asociación entre el apego parental y la dependencia emocional. En este sentido, Cerdán Fievez (2016) habla de la posibilidad de la "reparación" de apegos inseguros por la influencia de otras variables.

En el presente estudio también se encontró que la población que muestra apego inseguro ambivalente presenta una media más alta de relación con las diferentes dimensiones de dependencia emocional. Cavero (2008), afirma que el afecto y el control parental inestable percibido en la niñez, están relacionados positivamente con el apego ambivalente, el cual incrementa la necesidad de recibir constantemente muestras de afecto por parte de la pareja para sentirse amado y valorado. Así mismo, Castelló Blasco (2000) afirma que la dependencia emocional tiene una relación directa con el apego ansioso o ambivalente. Refiriéndose a los hallazgos de Alonso-Arbiol, Shaver y Yárnoz (2002) y Castelló Blasco (2013), quienes consideran que el apego ambivalente es la pauta predominante de las personas que presentan dependencia emocional pero no se aplica en caso inverso.

Valle y Moral (2018), tuvieron como propósito en su estudio, comprobar si existía una relación significativa entre los conceptos de dependencia emocional y apego adulto. En efecto, se obtuvo una correlación positiva tanto entre los estilos de apego y la existencia de dependencia emocional, como con su intensidad; aunque, contrario al presente estudio, fueron los jóvenes clasificados en el estilo de apego evitativo los de mayores niveles de dependencia emocional.

Los resultados de la presente investigación, van de acuerdo con lo afirmado por Castelló Blasco (2013), quien dice que las relaciones románticas son el área más relevante y en donde más se manifiestan las características del dependiente emocional, quien necesita estar siempre con su pareja, recibir aprobación, lograr relaciones exclusivas; además, tiene necesidad de expresiones de afecto constante, adopción de posiciones subordinadas y sumisas en las relaciones, con tal de retener a su pareja, hasta modificar sus planes por el miedo a la soledad. Ésta última, característica reseñada por Carvajal y Caro, (2009) quienes indican que la soledad puede manifestarse en los adolescentes a partir de dos contextos: en la relación parental y en la relación social a partir de los pares. Las características anteriormente mencionadas para el dependiente emocional, las asocia Bowlby (1993), en su teoría del apego parental con la" ansiedad de separación", en donde la persona con un sistema de apego ansioso activa exageradamente el sistema en situaciones que no percibe la cercanía con sus cuidadores.

\section{Conclusiones}

A partir de los hallazgos encontrados se concluye que todos los factores de dependencia emocional, están directamente relacionados con la ansiedad experimentada (apego inseguro) hacia la pareja, por lo tanto, sí existe una relación entre los estilos de apego parental y dependencia emocional en las relaciones románticas de los jóvenes que participaron.

En cuanto a las manifestaciones de dependencia emocional en las relaciones románticas y su relación con sexo y nivel socio económico, es posible concluir que sí hay una relación en cuanto al nivel socioeconómico y el sexo en las manifestaciones de dependencia emocional dentro de la relación románticas. La composición de la muestra permite obtener puntuaciones diferentes en los estilos de apego parental y dependencia emocional con relación a anteriores estudios que se realizaron en muestras puntuales en poblaciones específicas de Colombia.

Se sugiere que los estudios futuros obtengan una muestra más amplia por regiones, para evidenciar el contraste que los resultados puedan arrojar de acuerdo a los contextos culturales. Incluyendo, un estudio de la correlación de la dependencia emocional y el apego, a partir de una muestra aleatoria 
que permita determinar una visión de la realidad de Colombia. Finalmente, investigar en las diferentes etapas del ciclo vital, la correlación entre apego y dependencia emocional.

\section{Referencias}

Acosta, D., Amaya, P., \& De la Espriella, C. (2013). Estilos de apego parental y dependencia emocional en las relaciones románticas de los adolescentes. (Tesis de especialización). Universidad de la Sabana, Colombia. Recuperado de https://intellectum.unisabana. edu.co/bitstream/handle/10818/6773/125566. pdf?sequence $=1$ \&isAllowed $=y$

Ainsworth, M. D., Blehar, M. C., Waters, E., \& Wall, S. (2015). Patterns of Attachment: A Psychological Study of the Strange Situation. (Classic Edition). New York: Psychology Press and Routledge.

Armsden, G., \& Greenberg, M. (1987). The Inventory of Parent and Peer Attachment: Individual Differences and Their Relationship to Psychological Well-Being in Adolescence. Journal of Youth and Adolescence, 16(5), 42754. DOI: 10.1007/BF02202939

Blandón, A. I., \& López, L. M. (2016). Comprensiones sobre pareja en la actualidad: jóvenes en busca de estabilidad. Revista Latinoamericana de Ciencias Sociales, Niñez y Juventud, 14(1), 505517. DOI: 10.11600/1692715x.14134271014

Bowlby, J. (1980). Attachment and Loss. Loss sadness and depression. Volume III. New York: Perseus Books Group.

Bowlby, J. (1993). La separación afectiva. Barcelona. Paidós.

Bowlby, J. (1995). Una base segura. Aplicaciones Clínicas de una teoría del apego. Barcelona: Paidós.

Callejón-Chinchilla, M. D. \& Gila-Ordoñez, J. M. (2018) Necesidad de trabajar las relaciones de pareja saludable desde contextos socio edu- cativos. Sophia, 14(1), 31-38. DOI: 10.18634/ sophiaj.14v.1i.703

Carreras, M. A., Brizzio, A., Gonzalez, R., Mele, S., \& Casullo, M. (2008). Los estilos de apego en los vínculos románticos y no románticos. Estudio comparativo con adolescentes argentinos y españoles. Revista iberoamericana de diagnóstico y evaluación psicológica, 25(1), 107-124.

Carvajal-Carrascal, G. \& Caro-Castillo, C. (2010). Soledad en la adolescencia: análisis del concepto. Aquichan, 9(3), 281-296.

Castelló Blasco, J. (2000). Análisis del concepto "dependencia emocional", I Congreso Virtual de Psiquiatría, Conferencia 6-Cl-A: 52. Recuperado de http://files.psicodx-funccogpersonalidad.webnode.com.co/200000057412c14225d/dependencia\%20emocional.pdf

Castelló Blasco, J. (2005) Dependencia emocional: características y tratamiento (1. ${ }^{\mathrm{a}}$ ed.). España: Alianza Editorial.

Castelló Blasco, J. (2012). La superación de la dependencia emocional. Cómo impedir que el amor se convierta en un suplicio. España: Ediciones Corona Borealis.

Castelló Blasco, J. (2013). Dependencia emocional. Características y tratamiento ( $5 .^{a}$ ed.). España: Alianza Editorial.

Cavero, R. M. (2008). La relación de pareja, apego, dinámicas de interacción y actitudes amorosas: consecuencias sobre la calidad de la relación (Tesis doctoral). Universidad de Valencia. España. Recuperado de http://roderic.uv.es/ bitstream/handle/10550/15428/melero. pdf?sequence $=1$ \&isAllowed $=y$

Cerdán Fievez, S, (2016). Apego y relaciones románticas. (Tesis de pregrado). Universidad Pontificia Comillas, España. Recuperado de https://repositorio.comillas.edu/xmlui/ bitstream/handle/11531/13378/TFM000428. pdf?sequence $=1$ \&isAllowed $=y$ 
Cuervo Martínez, Á. (2010). Pautas de crianza y desarrollo socioafectivo en la infancia. Diversitas: Perspectivas en Psicología, 6(1), 111-121.

Feeney, J. A. \& Noller, P. (1990). Attachment style as a predictor of adult romantic relationships. Journal of Personality and Social Psychology, 58, 281-291.

Figueroa, J. G. \& Franzoni, J. (2011). Del hombre proveedor al hombre emocional: construyendo nuevos significados de la masculinidad entre varones mexicanos. En: F. Aguayo \& M. Sadler (Eds.), Masculinidades y Políticas Públicas: Involucrando Hombres en la equidad de género (pp. 64-81). Chile: Facultad de Ciencias Sociales, Universidad de Chile.

Furman, W. \& Collibee, C. (2018). The past is present: representations of parents, friends, and romantic partners predict subsequent romantic representations. Child Development, 89 (1), 188-204. DOI: 10.1111/cdev.12712.

Furman, W. \& Simon V.A (1999). Cognitive representations of romantic relationships In Furman W, Brown BB, y Feiring C (Eds.), The development of romantic relationships in adolescence (pp. 75-98). New York: Cambridge University Press.

García Molina, A., Enseñat Cantallops, A., Tirapu Ustárroz, J., \& Roig Rovira T. (2009). Maduración de la corteza prefrontal y desarrollo de las funciones ejecutivas durante los primeros cinco años de vida. Revista de Neurología, 48(8), 435-440.

Gómez Maquet, Y., Vallejo Zapata, V., Villada Zapata, J., \& Zambrano Cruz, R. (2011). Caracterización de lazos parentales en estudiantes de pregrado de la Universidad de Antioquia. Revista de Psicología Universidad de Antioquia, 1(1), 35-54.

Guzmán, M. \& Contreras, P. (2012). Estilos de Apego en Relaciones de Pareja y su Asociación con la Satisfacción Marital. Psykhe, 21(1), 69-82. DOI: 10.4067/S0718-22282012000100005
Hazan, C. \& Shaver, P. (1987). Romantic love conceptualized as an attachment process. Journal of Personality and Social Psychology, 52(3), 511524. DOI: 10.1037/0022-3514.52.3.511

Hernández, R., Fernández, C., \& Baptista, M. P. (2014). Metodología de la investigación. $6^{\mathrm{a}}$ edición. México: Editorial McGraw Hill.

Hernando-Gómez, A., Maraver-López, P. \& PazosGómez, M. (2016). Experiencias positivas y negativas en relaciones de pareja de jóvenes y adolescentes. Revista de Psicología, 25(2), 1-19. DOI: 10.5354/0719-0581.2016.44745

Laca Arocena, F. \& Mejía Ceballos, J. (2017). Dependencia emocional, consciencia del presente y estilos de comunicación en situaciones de conflicto con la pareja. Enseñanza $e$ Investigación en Psicología, 22(1), 66-75.

Lemos Hoyos, M. \& Londoño Arredondo, N. (2006). Construcción y validación del cuestionario de dependencia emocional en población colombiana. Acta Colombiana de Psicología, 9(2), 127-140.

Lemos, M., Jaller, C., González, A., Díaz, Z. \& De la Ossa, D. (2012). Perfil cognitivo de la dependencia emocional en estudiantes universitarios. Universitas Psychologica, 11(2), 1-10.

Lozano-Sapuy, L. P., Albarracín-Ángel, P. A., \& Vásquez-Amézquita, M. (2016). Apego parental y su relación con el apego romántico y la dependencia afectiva en 119 universitarios de la ciudad de Ibagué - Colombia. Revista de Psicología GEPU, 7(1), 8-39.

Machado, T. S., Dias-da-Costa, C., \& Da Silva, J. T. (2015). Vinculação aos pais e vinculação amorosa: esperança e satisfação com a vida. Revista de estudios e investigación en Psicología y Educación, 14, 001-005. DOI: 10.17979/ reipe.2015.0.14

Main, M. \& Solomon, J. (1990). Procedures for identifying infants as disorganized/disoriented during the Ainsworth Strange Situation. In M. T. Greenberg, D. Cicchetti, \& E. M. 
Cummings (Eds.), The John D. and Catherine T. MacArthur Foundation series on mental health and development. Attachment in the preschool years: Theory, research, and intervention (pp. 121-160). Chicago, IL, US: University of Chicago Press.

Martínez, V., Castañeiras, C., \& Posada M. (2011). Estilos de apego y autoconcepto: bases para una adolescencia positiva. Psicodebate. Psicología, Cultura y Sociedad, 1(11), 27-42. DOI: $10.18682 / p d . v 11 i 0.374$

Martínez, J., Fuertes, A., Orgaz, B., Vicario, I., \& González, E. (2014). Vínculos afectivos en la infancia y calidad en las relaciones de pareja de jóvenes adultos: el efecto mediador del apego actual. Anales de Psicología, 30(1), 211-220. DOI: 10.6018/analesps.30.1.135051

Masota, F. A., Sedano, A. R., \& de Soria, A. B. M. (2005). La familia, escuela de sociabilidad. Educación y educadores, 8, 173-186.

Moral, M. V., García, A., Cuetos, G., \& Sirvent, C. (2017). Violencia en el noviazgo, dependencia emocional y autoestima en adolescentes y jóvenes españoles. Revista Iberoamericana de Psicología y Salud, 8(2), 96-107. DOI: 10.23923/j. rips.2017.08.009

Moral, M.V., Sirvent, C., Ovejero, A., \& Cuetos, G. (2018). Dependencia emocional en las relaciones de pareja como Síndrome de Artemisa: modelo explicativo. Terapia psicológica, 36(3), 156-166.

Oliva, A. (2004). Estado actual de la teoría del apego. Revista de Psiquiatría y Psicología del Niño y del Adolescente, 4(1), 65-81.

Pardo, M. E., Pineda, S., Carrillo, S., \& Castro, J. (2006) Análisis psicométrico del inventario de apego con padres y pares en una muestra de adolescentes colombianos. Interamerican Journal of Psychology, 40(3), 289-302.

Quiñones, N., \& Wilson, J. (2013). Relación entre estilos parentales percibidos y dependencia emocional, en una muestra de adolescentes bogotanos. (Tesis de especialización). Universidad de la Sabana, Colombia. Recuperado de https://intellectum.unisabana. edu.co/bitstream/handle/10818/6772/125567. pdf?sequence $=1$ \&isAllowed $=y$

Reyes, V., \& Reidl, L. (2015). Apego, miedo, estrategias de afrontamiento y relaciones intrafamiliares en niños. Psicología y Salud, 25(1), 91-101.

Salgado, C., Álvarez, A., Hernández, V., Herrera, J., \& Sánchez, M. (2016). Dificultades interpersonales y sustancias psicoactivas en adolescentes. Revista Iberoamericana de las Ciencias de la Salud, 5(9), 51-67.

Schore, A. (2016). Affect Regulation and the Origin of the Self. New York: Routledge. DOI: 10.4324/9781315680019

Smith-Etxeberría, K., Ortiz-Barón, M. J. \& ApodacaUrquijo, P. (2014). Experiencias e interacciones de la familia de origen y su influencia en las relaciones afectivas de los adultos jóvenes. Apuntes de Psicología, 32(2), 127-136.

Soares, I. \& Dias, P. (2007). Apego y psicopatología en jóvenes y adultos: contribuciones recientes de la investigación. International Journal of Clinical and Health Psychology, 7, 177-195.

Sroufe, L. A. (2000). Early relationships and the development of children. Infant Mental Health Journal, 21(1-2), 67-74. DOI: 10.1002/ (SICI)1097-0355(200001/04)21:1/2<67::AIDIMHJ8>3.0.CO;2-2

Torres, A. \& Rodrigo, M. J. (2014). La influencia del apego y el autoconcepto en los problemas de comportamiento de los niños y niñas de familias en desventaja socioeconómica. Educatio Siglo XXI, 3(2), 255-278. DOI: 10.6018/j/194181

Valle, L. \& Moral, M. V., (2018). Dependencia emocional y estilo de apego adulto en relaciones de noviazgo en jóvenes españoles. Revista Iberoamericana de Psicología y salud, 9(1), 2741. https://doi.org/10.23923/j.rips.2018.01.013 
Vivona, J. (2000). Parental Attachment Styles of Late Adolescents Qualities of Attachment. Journal of Counseling Psychology, 47(3), 316329. DOI: 10.1037MX)22-0167.47.3.316
Yepes Stork, R. (1996). Fundamentos de Antropología. Ideal de la excelencia Humana. España. EUNSA. 